Real Analysis Exchange

Vol. 26(1), 2000/2001, pp. 133-156

Zoltán Buczolich*and Judit Nagy, Department of Analysis, Eötvös Loránd University, Kecskeméti u. 10-12 , 1053 Budapest, Hungary. e-mail:

buczo@cs.elte.hu and nmm@cs.elte.hu

\title{
HÖLDER SPECTRUM OF TYPICAL MONOTONE CONTINUOUS FUNCTIONS
}

\begin{abstract}
We compute the Hölder spectrum and the moment sums of the typical (in the sense of Baire category) monotone continuous function, $f$. We show that these functions are of multifractal nature, for example $\alpha$ equals the Hausdorff dimension of those points where such a function, $f$ is of exact Hölder class $\alpha \in[0,1]$. We also prove that $f$ "grows" only at those points where it has bad Hölder properties, that is, at those points where it is of Hölder class 0 . The upper moment $\operatorname{sum} \bar{\tau}_{f}(q)=\infty$ if $q<0$, $\bar{\tau}_{f}(q)=1-q$ if $0 \leq q \leq 1$, and $\bar{\tau}_{f}(q)=0$ if $1<q$. The lower moment $\operatorname{sum} \underline{\tau}_{f}(q)=1-q$ if $q<0, \underline{\tau}_{f}(q)=0$ if $0 \leq q \leq 1$, and $\underline{\tau}_{f}(q)=1-q$ if $1<q$.
\end{abstract}

\section{Introduction}

In this paper we investigate the multifractal (Hölder) properties of the typical (in the sense of Baire category) monotone continuous function defined on $[0,1]$. We denote the space of monotone increasing continuous functions equipped with the sup norm by $C_{\nearrow}[0,1]$. It is a separable, complete metric space, being a closed subspace of $C[0,1]$. If a property holds for a residual subset of $C_{\nearrow}[0,1]$, then we say that the typical function $f$ has this property.

In $[11,12]$ the differentiability properties of typical monotone, continuous functions were studied and it was shown that the typical monotone, continuous function, $f$ is a strictly monotone, increasing, singular, function and its derivative equals 0 wherever it exists. Of course, $f^{\prime}$ exists almost everywhere in $[0,1]$.

\footnotetext{
Key Words: typical monotone continuous function, moment sum, Hölder spectrum.
Mathematical Reviews subject classification: Primary 28A80, 26A16 Secondary: 58F12, $26 \mathrm{~A} 48$

Received by the editors June 28, 1999

* This author was supported by Grants FKFP 0192/1999 and Hungarian National Foundation for Scientific Research Grant No. T 016094
} 
Jaffard $[2,3,4,5]$ has studied the multifractal properties of several specific continuous functions. For these functions many interesting methods, for example, wavelets, Diophantine approximation etc. were used.

It is not difficult to verify that the typical continuous function on $[0,1]$ is quite uninteresting from our point of view; it does not have multifractal Hölder properties, in fact, it is Hölder class 0 everywhere in $[0,1]$. Hence it is much more interesting to look at the class of typical monotone, continuous functions which are of multifractal nature. By studying typical monotone, continuous functions we study typical continuous measures on $[0,1]$. We point out that typical measures on arbitrary separable metric spaces were considered by $[10,9]$, for example a typical measure typically has no local dimension.

We denote by $E_{\alpha}^{f}$ those points of $[0,1]$ where $f \in C_{\nearrow}[0,1]$ is of exact Hölder class $\alpha$ and by $E_{<\alpha}^{f}$ those points where $f$ is of Hölder class $\beta$ for any $\beta<\alpha$ and is not of Hölder class $\beta$ for any $\beta>\alpha$ (the detailed definitions will be given in the preliminaries section).

The main results of this paper are given in Theorem 6 . In assertions (i-ii) of Theorem 6 we show that $\operatorname{dim}_{H} E_{\alpha}^{f}=\operatorname{dim}_{H} E_{\leq \alpha}^{f}=\alpha$ holds for $\alpha \in[0,1]$, while $E_{\leq \alpha}^{f}=\emptyset$ for $\alpha>1$. This implies that for any $\alpha \in[0,1]$ there are "many points" where the typical $f \in C_{\nearrow}[0,1]$ function is of exact Hölder class $\alpha$. In assertion (vi) we will show that if $\alpha \neq 0$ then $f$ is not "growing" on the sets $\widehat{E}_{\leq \alpha}^{f} \supset E_{\alpha}^{f}$. In fact, all the growth of $f$ takes place on $E_{\leq 0}^{f}$, by this we mean that $\mu_{f}\left(E_{\leq 0}^{f}\right)=\mu_{f}([0,1])=f(1)-f(0)$, where $\mu_{f}$ is the Borel measure corresponding to $f$. Thus $\mu_{f}\left(\cup_{\alpha \neq 0} \widehat{E}_{\alpha}^{f}\right)=0$.

Studying multifractal properties quite often the moment sums, $\tau_{f}(q)$ (for the definition see (1) below), are used $[1,6,7]$ and in "lucky situations" the Legendre transform of $\tau_{f}(q)$ provides the spectrum of $f$. As one can expect the typical $f \in C_{\nearrow}[0,1]$ is not the "lucky case". In assertions (iii-v) of Theorem 6 we compute these moment sums. It turns out that for the typical $f \in C_{\nearrow}[0,1]$ $\tau_{f}(0)=1, \tau_{f}(1)=0$ and $\tau_{f}(q)$ does not exist for other values of $q$. If the upper moment sums are considered we have $\bar{\tau}_{f}(q)=\infty$ if $q<0, \bar{\tau}_{f}(q)=1-q$ if $0 \leq q \leq 1$ and $\bar{\tau}_{f}(q)=0$ if $1<q$. For the lower moment sums, $\underline{\tau}_{f}(q)$, we show that $\underline{\tau}_{f}(q)=1-q$ if $q<0, \underline{\tau}_{f}(q)=0$ if $0 \leq q \leq 1$ and $\underline{\tau}_{f}(q)=1-q$ if $1<q$. It might be worth to mention that an elementary computation shows that for $f(x)=x$ we have $\tau_{f}(q)=1-q$ for all $q \in \mathbb{R}$.

In Section 4 we present the main construction of this paper. This construction will provide the dense $G_{\delta}$ sets in $C_{\nearrow}[0,1]$ showing that certain properties are typical. The proof of Theorem 6 will be split into parts according to the different assertions made. 


\section{Preliminaries}

The integer part of the real number $x$; that is, the largest integer not exceeding $x$, will be denoted by $[x]$.

We use the notation $\lambda(A)$ for the Lebesgue measure of the measurable set $A \subset[0,1]$ while $\mathcal{H}^{s}(A)$ denotes its $s$-dimensional Hausdorff measure $[6,7]$.

The space of continuous and continuously differentiable functions on $[0,1]$ is denoted by $C[0,1]$ and by $C^{1}[0,1]$, respectively.

Definition 1. Given $\alpha \geq 0$ and a point $x_{0} \in[0,1]$ we say that the function $f$ is $C^{\alpha}\left(x_{0}\right)$ if there exists a polynomial $P$ of degree at most $[\alpha]$ and a constant $C>0$ such that $\left|f(x)-P\left(x-x_{0}\right)\right| \leq C\left|x-x_{0}\right|^{\alpha}$ for all $x \in[0,1]$. The Hölder exponent of $f$ at $x_{0}$ is defined by

$$
h_{f}\left(x_{0}\right)=\sup \left\{\alpha: f \text { is } C^{\alpha}\left(x_{0}\right)\right\} .
$$

Set

$$
E_{\leq \alpha}^{f}=\left\{x_{0}: h_{f}\left(x_{0}\right) \leq \alpha\right\} \text { and } E_{\alpha}^{f}=\left\{x_{0}: h_{f}\left(x_{0}\right)=\alpha \text {, and } f \text { is } C^{\alpha}\left(x_{0}\right)\right\} .
$$

The set $E_{\alpha}^{f}$ denotes the set of those points where $f$ is of "exact" Hölder class $\alpha$. It is clear that $E_{\alpha}^{f} \subset E_{\leq \alpha}^{f} \backslash \cup_{\beta<\alpha} E_{\leq \beta}^{f}=\widehat{E}_{\alpha}^{f}$. However this inclusion is proper; for example if we take $g(x)=-\log (x / 10) \cdot \sqrt{x / 10}$ for $x \in(0,1]$ and $g(0) \stackrel{\text { def }}{=} 0$, then $g \in C_{\nearrow}[0,1], h_{g}(0)=1 / 2$, but $g$ is not $C^{1 / 2}(0)$.

To compute Hausdorff dimensions we will use Proposition 2.2 of [7] (see also Proposition 4.9 of [6]).

Proposition 1. Let $E \subset \mathbb{R}^{n}$ be a Borel set, let $\mu$ be a finite Borel measure on $\mathbb{R}^{n}$ and $0<c<\infty$.

(a) If $\lim \sup _{r \rightarrow 0+} \mu(B(x, r)) / r^{s} \leq c$ for all $x \in E$, then $\mathcal{H}^{s}(E) \geq \mu(E) / c$.

(b) If $\lim \sup _{r \rightarrow 0+} \mu(B(x, r)) / r^{s} \geq c$ for all $x \in E$, then $\mathcal{H}^{s}(E) \leq 2^{s} \mu(E) / c$.

\section{Elementary Lemmas for the Moment Sums}

Given a monotone increasing continuous function $g:[0,1] \rightarrow \mathbb{R}$ we denote by $\mu_{g}$ the Borel measure corresponding to $g$; that is, for which $\mu_{g}((a, b))=$ $g(b)-g(a)$ for each interval $(a, b)$.

Set

$$
\tau_{g, j}(q)=\frac{1}{j \log 2} \log \sum_{k=0}^{2^{j}-1}\left(\mu_{g}\left(\left[\frac{k}{2^{j}}, \frac{k+1}{2^{j}}\right]\right)\right)^{q} \text { and } \tau_{g}(q)=\lim _{j \rightarrow \infty} \tau_{g, j}(q) .
$$


If the latter limit does not exist, we use $\bar{\tau}_{g}(q)=\lim \sup _{j \rightarrow \infty} \tau_{g, j}(q)$ and $\underline{\tau}_{g}(q)=$ $\liminf _{j \rightarrow \infty} \tau_{g, j}(q)$.

It is not difficult to see that $\tau_{c g}(q)=\tau_{g}(q)$ holds for any $c>0$; so there is no problem if the finite measure $\mu_{g}$ is not a probability measure as long as $g$ is a nonconstant increasing function, actually, when we compute moment sums we can assume that $\mu_{g}$ is a probability measure.

Lemma 2. If $g \in C^{1}[0,1]$ and $g^{\prime}>0$, then for every $\epsilon>0$ there exists $K>0$ such that $\tau_{g, j}(q) \geq 1-q-\epsilon$ for all $j \geq K, q \in[0,1]$.

Proof. Using the continuity of $g^{\prime}>0$ we can choose $\varrho_{1}$ such that $g^{\prime}>\varrho_{1}>0$. Using the mean value theorem for $g$ from the above estimates we infer

$$
\mu_{g}\left(\left[\frac{k}{2^{j}}, \frac{k+1}{2^{j}}\right]\right) \geq \varrho_{1} \frac{1}{2^{j}} .
$$

Then

$$
\tau_{g, j}(q) \geq \frac{1}{j \log 2} \log \left(2^{j}\left(\frac{\varrho_{1}}{2^{j}}\right)^{q}\right)=1-q+\frac{q \log \varrho_{1}}{j \log 2} .
$$

This proves the lemma.

Lemma 3. For any $q \in[0,1]$ and $g \in C_{\nearrow}[0,1]$ we have $\bar{\tau}_{g}(q) \leq 1-q$ and $\underline{\tau}_{g}(q) \geq 0$.

Proof. Set $\mu_{k, j}=\mu_{g}\left(\left[\frac{k}{2^{j}}, \frac{k+1}{2^{j}}\right]\right)$. If $q=0$, then $\sum_{k=0}^{2^{j}-1} \mu_{k, j}^{0}=2^{j}$ and hence $\bar{\tau}_{g}(0)=\tau_{g}(0)=1$. In the sequel, we assume $q \in(0,1]$. Obviously, $\sum_{k=0}^{2^{j}-1} \mu_{k, j}=$ $g(1)-g(0) \stackrel{\text { def }}{=} G_{0}>0$. Since $\mu_{k, j} \geq 0$ we can use some well-known inequalities between means of nonnegative numbers and obtain

$$
\left(\frac{\sum_{k=0}^{2^{j}-1} \mu_{k, j}^{q}}{2^{j}}\right)^{\frac{1}{q}} \leq \frac{\sum_{k=0}^{2^{j}-1} \mu_{k, j}}{2^{j}}=\frac{G_{0}}{2^{j}} .
$$

This implies $\sum_{k=0}^{2^{j}-1} \mu_{k, j}^{q} \leq G_{0}^{q} 2^{j(1-q)}$ and an easy calculation implies $\bar{\tau}_{g}(q) \leq$ $1-q$.

Using that $\tau_{c g}=\tau_{g}$ for any $c>0$ we can assume $\mu_{g}([0,1])=G_{0}=1$. Then $\mu_{k, j}^{q} \geq \mu_{k, j}$ and hence $\sum_{k=0}^{2^{j}-1} \mu_{k, j}^{q} \geq \sum_{k=0}^{2^{j}-1} \mu_{k, j}=G_{0}=1$. Thus $\tau_{g, j}(q) \geq 0$ and hence $\underline{\tau}_{g}(q) \geq 0$.

The next two lemmas are the $q>1$ versions of the previous two ones. Their proofs are analogous so we omit some details. 
Lemma 4. If $g \in C^{1}[0,1]$ and $g^{\prime} \geq 0$, then for every $\epsilon>0$ there exists $K>0$ such that $\tau_{g, j}(q) \leq 1-q+\epsilon$ for all $j \geq K, q \in[1,1 / \epsilon]$.

Proof. Using the continuity of $g^{\prime}>0$ we can choose $\varrho_{2}$ such that $\varrho_{2}>g^{\prime}$. By the mean value theorem we infer

$$
\mu_{g}\left(\left[\frac{k}{2^{j}}, \frac{k+1}{2^{j}}\right]\right) \leq \varrho_{2} \frac{1}{2^{j}} .
$$

Then

$$
\tau_{g, j}(q) \leq \frac{1}{j \log 2} \log \left(2^{j}\left(\frac{\varrho_{2}}{2^{j}}\right)^{q}\right)=1-q+\frac{q \log \varrho_{2}}{j \log 2} .
$$

Lemma 5. For any $q>1$ and $g \in C_{\nearrow}[0,1]$ we have $\underline{\tau}_{g}(q) \geq 1-q$ and $\bar{\tau}_{g}(q) \leq 0$.

Proof. Define $\mu_{k, j}$ and $G_{0}$ as in the proof of Lemma 2. From $q>1$ it follows that we have the reversed inequality in (2); that is,

$$
\left(\frac{\sum_{k=0}^{2^{j}-1} \mu_{k, j}^{q}}{2^{j}}\right)^{\frac{1}{q}} \geq \frac{G_{0}}{2^{j}} .
$$

This implies $\sum_{k=0}^{2^{j}-1} \mu_{k, j}^{q} \geq G_{0}^{q} 2^{j(1-q)}$ and hence $\underline{\tau}_{g}(q) \geq 1-q$.

As in Lemma 2 we can assume $\mu_{g}([0,1])=1$ and using $\mu_{k, j}^{q} \leq \mu_{k, j}$ we obtain $\sum_{k=0}^{2^{j}-1} \mu_{k, j}^{q} \leq 1$ which implies $\tau_{g, j}(q) \leq 0$ and $\bar{\tau}_{g}(q) \leq 0$.

\section{The Main Construction}

Assume $m \in \mathbb{N}$ is given. We choose a countable dense subset, $\left\{f_{n}\right\}$, in $C_{\nearrow}[0,1]$, such that each $f_{n}$ is continuously differentiable and $f_{n}^{\prime}>0$ on $[0,1]$. Using Lemmas 2 and 4 with $\epsilon=1 / n$ choose $K_{n}>n$ such that for all $q \in[0,1]$ and $j \geq K_{n}$ we have

$$
\tau_{f_{n}, j}(q) \geq 1-q-\frac{1}{n}
$$

and for all $q \in[1, n]$ and $j \geq K_{n}$ we have

$$
\tau_{f_{n}, j}(q) \leq 1-q+\frac{1}{n}
$$


Using the uniform continuity of $f_{n}$ choose and fix $N_{n} \geq K_{n}$ such that

$$
f_{n}\left(\frac{k+1}{2^{N_{n}}}\right)-f_{n}\left(\frac{k}{2^{N_{n}}}\right)=\mu_{f_{n}}\left(\left[\frac{k}{2^{N_{n}}}, \frac{k+1}{2^{N_{n}}}\right]\right)<\frac{1}{2 n}
$$

holds for $k=0, \ldots, 2^{N_{n}}-1$.

We will choose a large integer $\nu_{n, m}>\max \left\{N_{n}^{2}, m\right\}$ to be determined later. Set

$$
\begin{gathered}
h_{n, m}^{*}=\frac{1}{4^{n} 2^{m \nu_{n, m}}}<\frac{1}{4^{n}}, \quad h_{n, m}=\frac{h_{n, m}^{*}}{2^{\nu_{n, m}}}, \\
\delta_{n, m}=\left(h_{n, m}\right)^{2 m} \frac{1}{4}<\frac{1}{2} \frac{1}{2^{\nu_{n, m}}}, \quad P(k, n, m)=\frac{k}{2^{\nu_{n, m}}}+\delta_{n, m},
\end{gathered}
$$

where $k \in\left\{0, \ldots, 2^{\nu_{n, m}}-1\right\}$. In some applications of our construction we will use different values for $h_{n, m}^{*}$. However for all these values we will have $0<$ $h_{n, m}^{*} \leq 1 / 4^{n} \leq 1 / 4 n$. Observe that $h_{n, m}^{*}, h_{n, m}, \delta_{n, m}$ and $P(k, n, m)$ are all dyadic rationals.

Set

$$
W_{n, m}=\bigcup_{k=0}^{2^{\nu_{n}, m}-1}\left(\frac{k}{2^{\nu_{n, m}}}, P(k, n, m)\right)
$$

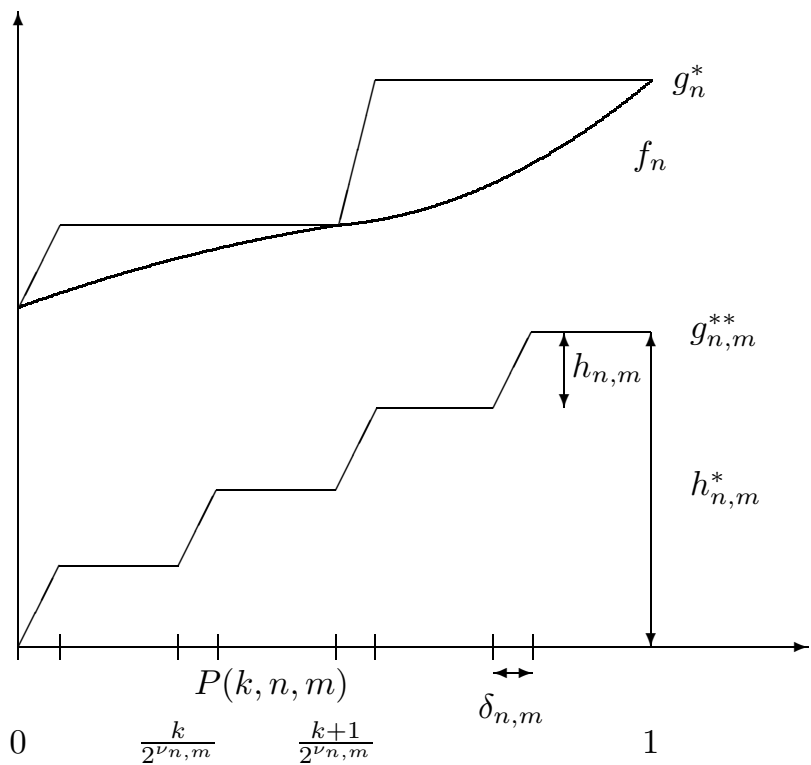

Next we define the auxiliary function $g_{n}^{*}(x)=f_{n}\left(\left(k^{\prime}+1\right) / 2^{N_{n}}\right)$ if $x \in$ $\left(k^{\prime} / 2^{N_{n}},\left(k^{\prime}+1\right) / 2^{N_{n}}\right] \backslash W_{n, m},\left(k^{\prime}=0, \ldots, 2^{N_{n}}-1\right)$ and $g_{n}^{*}(0)=f_{n}(0)$. On 
$W_{n, m}$ we define $g_{n}^{*}$ so that it is continuous on $[0,1]$ and is linear on each interval $\left(k / 2^{\nu_{n, m}}, P(k, n, m)\right)$.

Set $g_{n, m}^{* *}(x)=(k+1) h_{n, m}$ if $x \in\left(k / 2^{\nu_{n, m}},(k+1) / 2^{\nu_{n, m}}\right] \backslash W_{n, m},(k=$ $\left.0, \ldots, 2^{\nu_{n, m}}-1\right)$ and $g_{n, m}^{* *}(0)=0$. On $W_{n, m}$ we define $g_{n, m}^{* *}$ so that it is continuous on $[0,1]$ and is linear on each interval $\left(k / 2^{\nu_{n, m}}, P(k, n, m)\right)$.

Finally we put $g_{n, m}(x)=g_{n}^{*}(x)+g_{n, m}^{* *}(x)$.

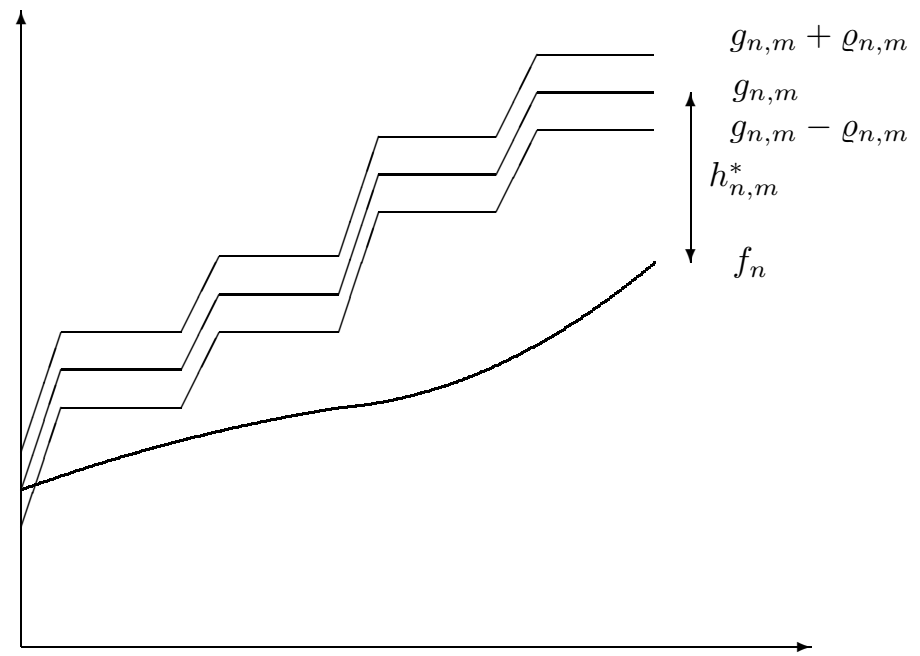

By (5)

$$
\left|f_{n}(x)-g_{n}^{*}(x)\right|<\frac{1}{2 n} \text { and } 0 \leq g_{n, m}^{* *}(x) \leq g_{n, m}^{* *}(1)=h_{n, m}^{*} .
$$

We also have

$$
\left|f_{n}(x)-g_{n, m}(x)\right| \leq\left|f_{n}(x)-g_{n}^{*}(x)\right|+h_{n, m}^{*} \leq \frac{3}{4 n}
$$

Using

$$
\varrho_{n, m}=\frac{\left(h_{n, m}\right)^{m}}{8}<\frac{h_{n, m}}{8}
$$

we set $G_{n, m}=B\left(g_{n, m}, \varrho_{n, m}\right)$ and $H_{m}=\cup_{n=m}^{\infty} G_{n, m}$. It is clear that $H_{m}$ is open in $C_{\nearrow}[0,1]$ and using the density of the functions $\left\{f_{n}\right\}$ and (9) it is easy to see that $H_{m}$ is dense. To prove the main result of the paper we will use the residual set $\mathcal{F}^{*}=\cap_{m=1}^{\infty} H_{m}$. 


\section{$5 \quad$ Main Results}

The purpose of this paper is to prove the following theorem.

Theorem 6. There is a residual subset $\mathcal{F} \subset C_{\nearrow}[0,1]$ such that for any $f \in \mathcal{F}$ we have

(i) $\widehat{E}_{\alpha}^{f}=\emptyset$ for $\alpha>1$;

(ii) $\operatorname{dim}_{H} E_{\alpha}^{f}=\operatorname{dim}_{H} \widehat{E}_{\alpha}^{f}=\operatorname{dim}_{H} E_{\leq \alpha}^{f}=\alpha$ for all $\alpha \in[0,1]$;

(iii) $\bar{\tau}_{f}(q)=1-q$ and $\underline{\tau}_{f}(q)=0$ for $0<q \leq 1$, and $\bar{\tau}_{f}(0)=\underline{\tau}_{f}(0)=\tau_{f}(0)=$ 1 ;

(iv) $\bar{\tau}_{f}(q)=0$ and $\underline{\tau}_{f}(q)=1-q$ for $1<q$;

(v) $\bar{\tau}_{f}(q)=\infty$ and $\underline{\tau}_{f}(q)=1-q$ for $q<0$;

(vi) $\mu_{f}\left(E_{\leq 0}^{f}\right)=f(1)-f(0)$; that is, $\mu_{f}\left([0,1] \backslash E_{\leq 0}^{f}\right)=0$.

We will split the proof of the theorem according to the assertions (i-vi). It is also clear that if for each assertion we can find a suitable dense $G_{\delta}$ set in $C_{\nearrow}[0,1]$, then the intersection of these sets will provide $\mathcal{F}$.

\subsection{Assertion (i)}

Proof. Recall that a monotone function is differentiable almost everywhere and by $[11,12]$ there is a residual, dense $G_{\delta}$ subset, $\mathcal{F}_{0}$ of $C_{\nearrow}[0,1]$ such that $f^{\prime}(x)=0$ at all points where $f^{\prime}$ exists. We use a slightly modified version of the main construction, in equation (6) we set $h_{n, m}^{*}=\frac{1}{4^{n}}$. With these values of $h_{n, m}^{*}$ the sets $H_{m}$ will still remain dense and open in $C_{\nearrow}[0,1]$. By choosing $\nu_{n, m} \geq m^{2}$ sufficiently large we can assume that

$$
h_{n, m}=\frac{h_{n, m}^{*}}{2^{\nu_{n, m}}}=\frac{1}{4^{n} 2^{\nu_{n, m}}}>2\left(\frac{1}{2^{\nu_{n, m}}}\right)^{1+\frac{1}{m}} .
$$

Denote by $\mathcal{F}_{1}=\cap_{m=1}^{\infty} H_{m}$ the set obtained by the main construction and suppose that $f \in \mathcal{F}_{(i)} \stackrel{\text { def }}{=} \mathcal{F}_{1} \cap \mathcal{F}_{0}$.

Proceeding towards a contradiction assume $1<\alpha<2, x_{0} \in[0,1]$ and $f$ is $C^{\alpha}\left(x_{0}\right)$. Then

$$
\left|f(x)-\left(a_{0}+a_{1}\left(x-x_{0}\right)\right)\right| \leq C\left|x-x_{0}\right|^{\alpha}
$$

and, clearly, $a_{0}=f\left(x_{0}\right), f^{\prime}\left(x_{0}\right)$ exists and $a_{1}=f^{\prime}\left(x_{0}\right)=0$ where we also used that $f \in \mathcal{F}_{0}$. Hence

$$
\left|f(x)-f\left(x_{0}\right)\right| \leq C\left|x-x_{0}\right|^{\alpha}
$$


Fix $m>2$ such that $\alpha>1+\frac{2}{m}$ and $C<m$. Choose $\delta>0$ such that $m|r|^{1 / m}<1$ holds for $0<|r|<\delta$; hence

$$
C|r|^{\alpha}<m|r|^{\alpha}<m|r|^{1+\frac{2}{m}}<|r|^{1+\frac{1}{m}}
$$

is also true. From $f \in \mathcal{F}_{1}$ it follows that there exists $n \geq m$ such that $f \in G_{n, m}$. We can also suppose that $\nu_{n, m}$ is so large that $2^{-\nu_{n, m}}<\delta$. Set $r=1 / 2^{\nu_{n, m}}$ if $x_{0}+r \in[0,1]$, otherwise set $r=-1 / 2^{\nu_{n, m}}$. Clearly,

$$
\left|g_{n, m}^{* *}\left(x_{0}+r\right)-g_{n, m}^{* *}\left(x_{0}\right)\right|=h_{n, m} \text { and } g_{n}^{*}, g_{n, m}^{* *} \text { are increasing; }
$$

hence

$$
\left|g_{n, m}\left(x_{0}+r\right)-g_{n, m}\left(x_{0}\right)\right| \geq h_{n, m} .
$$

Using $f \in B\left(g_{n, m}, \varrho_{n, m}\right)$ and $\varrho_{n, m}<h_{n, m} / 8$ we obtain

$$
\left|f\left(x_{0}+r\right)-f\left(x_{0}\right)\right|>\left|g_{n, m}\left(x_{0}+r\right)-g_{n, m}\left(x_{0}\right)\right|-2 \varrho_{n, m}>\frac{3}{4} h_{n, m} .
$$

Therefore by recalling (11) and (12)

$$
\frac{3}{4} h_{n, m}<\left|f\left(x_{0}+r\right)-f\left(x_{0}\right)\right| \leq C|r|^{\alpha}<|r|^{1+\frac{1}{m}}=\left(\frac{1}{2^{\nu_{n, m}}}\right)^{1+\frac{1}{m}}<\frac{h_{n, m}}{2},
$$

a contradiction.

We showed that for $1<\alpha<2$ there is no $x_{0} \in[0,1]$ for which $f$ is $C^{\alpha}\left(x_{0}\right)$. If $f$ is $C^{\beta}\left(x_{0}\right)$ and $0<\alpha<\beta$, then $f$ is $C^{\alpha}\left(x_{0}\right)$ as well. Thus we showed that $h_{f}\left(x_{0}\right) \leq 1$ for any $f \in \mathcal{F}_{(i)}$ and $x_{0} \in[0,1]$.

\section{$5.2 \quad$ Assertion (ii)}

Before turning to this assertion we state and prove a lemma.

Lemma 7. Assume $g \in C_{\nearrow}[a, b]$ and

$$
U_{g}=\{x \in[a, b]: \exists y \in[a, b] \text { such that }|g(y)-g(x)| \geq|y-x|\} .
$$

Then $\lambda\left(U_{g}\right) \leq 2(g(b)-g(a))$.

Proof. Set

$$
U_{1}=\{x \in[a, b]: \exists y \in(x, b] \text { such that } g(y)>g(x)+(y-x)\}
$$

and

$$
U_{2}=\{x \in[a, b]: \exists y \in[a, x) \text { such that } g(y)<g(x)+(y-x)\} .
$$


Clearly, $U_{g}=U_{1} \cup U_{2}$. We will show that $\lambda\left(U_{1}\right) \leq g(b)-g(a)$, the proof for $U_{2}$ is similar.

Letting $g_{0}(x)=g(x)-x$ we have

$$
U_{1}=\left\{x \in[a, b]: \exists y \in(x, b] \text { such that } g_{0}(y)>g_{0}(x)\right\} .
$$

Set $g_{1}(x)=\max \left\{g_{0}(t): t \in[x, b]\right\}$. Then $g_{1}(b)=g_{0}(b), g_{1}(a) \geq g_{0}(a)$ and $g_{1}^{\prime}(b) \geq-1$ where this derivative exists.

It is easy to see that $g_{1}(x)$ is monotone decreasing and hence (by using Theorem 2.9 .19 of $[8]$ )

$$
\int_{[a, b]} g_{1}^{\prime} \leq g_{1}(b)-g_{1}(a) \leq g_{0}(b)-g_{0}(a)=g(b)-g(a)+(a-b) .
$$

On the other hand $g_{1}^{\prime}=0$ almost everywhere on $U_{1}$ and hence

$$
\int_{[a, b]} g_{1}^{\prime} \geq \int_{[a, b] \backslash U_{1}}(-1)=(a-b)+\lambda\left(U_{1}\right) .
$$

By the above two displayed inequalities it follows that $\lambda\left(U_{1}\right) \leq g(b)-g(a)$.

Now we verify assertion (ii).

Proof. Any $f \in \mathcal{F}_{1}$ is almost everywhere differentiable, and hence it is $C^{1}\left(x_{0}\right)$ at almost every $x_{0} \in[0,1]$. This and assertion (i) $\operatorname{imply}_{\operatorname{dim}}\left(E_{1}^{f}\right)=1$.

Fix $0<\alpha<1$ and $1>\beta>\alpha$. Next we show that $\operatorname{dim}_{H} E_{<\alpha}^{f} \leq \alpha$. Actually, this is valid for any $f \in C_{\nearrow}[0,1]$ and follows easily from Proposition 1. If $x_{0} \in E_{\leq \alpha}^{f}$, then $f$ is not $C^{\beta}\left(x_{0}\right)$ and hence there exists $x_{n} \rightarrow x_{0}$ such that

$$
\left|f\left(x_{n}\right)-f\left(x_{0}\right)\right| \geq\left|x_{n}-x_{0}\right|^{\beta} .
$$

Letting $r_{n}=\left|x_{n}-x_{0}\right|$ we have

$$
\frac{\mu_{f}\left(B\left(x_{0}, r_{n}\right)\right)}{r_{n}^{\beta}} \geq 1
$$

This implies $\lim \sup _{r \rightarrow 0+} \mu_{f}\left(B\left(x_{0}, r\right)\right) / r^{\beta} \geq 1$. From Proposition 1 it follows that $\mathcal{H}^{\beta}\left(E_{\leq \alpha}^{f}\right) \leq 2^{\beta} \mu_{f}\left(E_{\leq \alpha}^{f}\right)<\infty$ and hence $\operatorname{dim}_{H} E_{\leq \alpha}^{f} \leq \beta$. Since this holds for any $\beta>\alpha$ we obtain $\operatorname{dim}_{H} E_{\leq \alpha}^{f} \leq \alpha$.

We turn to the much more difficult task to verify that $\operatorname{dim}_{H} E_{\alpha}^{f} \geq \alpha$, where $0<\alpha<1$ is fixed. Since $E_{\alpha}^{f} \subset E_{\leq \alpha}^{f}$ this will imply $\operatorname{dim}_{H} E_{\alpha}^{f}=\operatorname{dim}_{H} E_{\leq \alpha}^{f}=$ $\alpha$. We will use the main construction again with $h_{n, m}^{*}=1 / 4^{n}$ and assume 
that $f \in \mathcal{F}_{1}=\cap_{m=1}^{\infty} H_{m}$. This means that for each $m$ there exists $n(m) \geq m$ such that $f \in \cap_{m=1}^{\infty} G_{n(m), m}$. We will choose a suitable subsequence $m_{\ell} \geq \ell$. For ease of notation we let $G_{\ell}=G_{n\left(m_{\ell}\right), m_{\ell}}, N_{\ell}=N_{n\left(m_{\ell}\right)}, \nu_{\ell}=\nu_{n\left(m_{\ell}\right), m_{\ell}}$, $h_{\ell}=h_{n\left(m_{\ell}\right), m_{\ell}}, \delta_{\ell}=\delta_{n\left(m_{\ell}\right), m_{\ell}}, \varrho_{\ell}=\varrho_{n\left(m_{\ell}\right), m_{\ell}}$, and $P_{\ell}(k)=\frac{k}{2^{N_{\ell}}}+\delta_{\ell}$ for $k=0, \ldots, 2^{N_{\ell}}-1$. Observe that each of the above numbers is a dyadic rational.

We choose $h_{\ell}^{\prime}$ such that it is a dyadic rational and

$$
2^{\ell-1} h_{\ell}^{1 / \alpha} \leq h_{\ell}^{\prime} \leq 2^{\ell} h_{\ell}^{1 / \alpha} .
$$

Set $h_{\ell}^{\prime \prime}=h_{\ell}^{\prime} / 2^{\ell}$. Thus

$$
\frac{h_{\ell}^{1 / \alpha}}{2} \leq h_{\ell}^{\prime \prime} \leq h_{\ell}^{1 / \alpha}
$$

We assume that the subsequence $m_{\ell}$ is sufficiently rapidly increasing; namely, $2^{m_{\ell+1}}$ exceeds the denominator of the above numbers at "level $\ell$ ". This assumption will be convenient; for example, this way the intervals used in the definitions of $g_{\ell+1}^{*}=g_{n\left(m_{\ell+1}\right)}^{*}$ and $g_{\ell+1}^{* *}=g_{n\left(m_{\ell+1}\right), m_{\ell+1}}^{*}$, will belong to a subdivision of the intervals occurring at "level $\ell$ ".

Next we define closed sets $F_{\ell}^{\prime}$ and $F_{\ell}$ and using their intersection we define some Cantor type sets. Using Proposition 1 we will estimate the Hausdorff dimension of these sets. Furthermore we will also obtain information about the Hölder properties of $f$ on these sets. Put

$$
F_{\ell}^{\prime}=\bigcup_{k=0}^{2^{\nu}}\left[P_{\ell}(k)+h_{\ell}^{\prime \prime}, P_{\ell}(k)+h_{\ell}^{\prime}\right] \text { and } F_{\ell}=\bigcap_{\ell^{\prime} \leq \ell} F_{\ell}^{\prime} .
$$

We also let $F_{\infty}=\cap_{\ell=1}^{\infty} F_{\ell}$.

It is clear that if we choose the sequence $m_{\ell}$ to be sufficiently rapidly increasing, then each $F_{\ell}$ consists of $\kappa(\ell)>2^{\ell}$ many intervals of the form $J_{\ell, k}=\left[P_{\ell}(k)+h_{\ell}^{\prime \prime}, P_{\ell}(k)+h_{\ell}^{\prime}\right]$. By repeated subdivision ([7] pp. 9-10) we define a Borel probability measure, $\mu_{\alpha}$ with support on $F_{\infty}$ by setting $\mu_{\alpha}\left(J_{\ell, k}\right)=$ $1 / \kappa(\ell)$, that is, we assign equal weight to each interval $J_{\ell, k}$.

Assume $\ell \geq 2$. Choose a component of $F_{\ell-1}$. This component is a closed interval of length $h_{\ell-1}^{\prime \prime \prime} \stackrel{\text { def }}{=} h_{\ell-1}^{\prime}-h_{\ell-1}^{\prime \prime}$ and $F_{\ell}^{\prime}$ has one subinterval in each interval $\left[k / 2^{\nu_{\ell}},(k+1) / 2^{\nu_{\ell}}\right]$. (We assumed that $m(\ell)$ is increasing sufficiently fast; hence from $h_{\ell-1}^{\prime \prime \prime}$ being at "level $\ell-1$ " it follows that it is an integer multiple of $1 / 2^{\nu_{\ell}}$.) Then $\kappa(\ell) \geq h_{\ell-1}^{\prime \prime \prime} 2^{\nu_{\ell}}$ and hence

$$
\mu_{\alpha}\left(J_{\ell, k}\right) \leq \frac{1}{h_{\ell-1}^{\prime \prime \prime} 2^{\nu_{\ell}}} .
$$


Our next purpose is to show that for all $x \in F_{\infty}$ we have

$$
\limsup _{r \rightarrow 0+} \frac{\mu_{\alpha}(B(x, r))}{r^{\alpha-\epsilon}}<1
$$

for all $\epsilon \in(0, \alpha)$. We consider $r \in\left(1 / 2^{\nu \ell+1}, 1 / 2^{\nu \ell}\right]$ for each $\ell=2,3, \ldots$. We split each such case into two subcases

I. $h_{\ell}^{\prime}<r \leq 1 / 2^{\nu_{\ell}}$;

II. $1 / 2^{\nu_{\ell+1}}<r \leq h_{\ell}^{\prime}$

Assuming $\epsilon \in(0, \alpha)$ is fixed first we consider subcase I. In this case $B(x, r)$ can intersect at most three different $J_{\ell, k}$ and using (13) and (6)

$$
\begin{aligned}
\frac{\mu_{\alpha}(B(x, r))}{r^{\alpha-\epsilon}} & \leq \frac{\frac{3}{h_{\ell-1}^{\prime \prime \prime} 2^{\nu_{\ell}}}}{\left(h_{\ell}^{\prime}\right)^{\alpha-\epsilon}} \leq \frac{\frac{3}{h_{\ell-1}^{\prime \prime \prime} 2^{\nu}}}{2^{(\ell-1)(\alpha-\epsilon)} h_{\ell}^{(\alpha-\epsilon) / \alpha}} \\
& =\frac{3 \cdot 2^{(\epsilon-\alpha)(\ell-1)}}{h_{\ell-1}^{\prime \prime \prime}\left(h_{\ell}^{*}\right)^{(\alpha-\epsilon) / \alpha}} 2^{\nu_{\ell}\left(\frac{\alpha-\epsilon}{\alpha}-1\right)} \leq \frac{3 \cdot 4^{m_{\ell}}}{h_{\ell-1}^{\prime \prime \prime}} 2^{\nu_{\ell}\left(\frac{\alpha-\epsilon}{\alpha}-1\right)} \\
& <\frac{3 \cdot 4^{m_{\ell}}}{h_{\ell-1}^{\prime \prime \prime}} 2^{m_{\ell}^{2}\left(\frac{\alpha-\epsilon}{\alpha}-1\right)}<\frac{1}{4}
\end{aligned}
$$

where the last inequality holds when $m_{\ell}$ is chosen to be sufficiently large.

Subcase II. In this case $B(x, r)$ can intersect less than $\left(2 r / 2^{-\nu_{\ell+1}}\right)+2 \leq$ $4 r 2^{\nu_{\ell+1}}$ many components of $F_{\ell+1}$, each of $\mu_{\alpha}$ measure $\mu_{\alpha}\left(J_{\ell, k}\right) 2^{-\nu_{\ell+1}} / h_{\ell}^{\prime \prime \prime}$. By (15)

$$
\mu_{\alpha}(B(x, r)) \leq \frac{4 r}{h_{\ell}^{\prime \prime \prime}} \mu_{\alpha}\left(J_{\ell, k}\right) \leq 4 \frac{r}{h_{\ell}^{\prime}} \frac{1}{h_{\ell-1}^{\prime \prime \prime} 2^{\nu_{\ell}}} .
$$

Thus

$$
\begin{aligned}
\frac{\mu_{\alpha}(B(x, r))}{r^{\alpha-\epsilon}} & \leq \frac{4 \frac{r}{h_{\ell}^{\prime}} \frac{1}{h_{\ell-1}^{\prime \prime \prime} 2^{\nu \ell}}}{\left(\frac{r}{h_{\ell}^{\prime}} h_{\ell}^{\prime}\right)^{\alpha-\epsilon}} \\
& =4\left(\frac{r}{h_{\ell}^{\prime}}\right)^{1-(\alpha-\epsilon)} \frac{1}{\frac{h_{\ell-1}^{\prime \prime \prime} 2^{\nu} \ell}{\left(h_{\ell}^{\prime}\right)^{\alpha-\epsilon}}} \leq 4 \frac{\frac{1}{\left(h_{\ell-1}^{\prime \prime \prime} 2^{\nu^{\prime}}\right.}}{\left(h_{\ell}^{\prime}\right)^{\alpha-\epsilon}}<1
\end{aligned}
$$

where in the last line we used an estimate similar to the one used in subcase I. Hence for all $x \in F_{\infty}$ we have (16).

Next we investigate the Hölder properties of $f$ at points of $F_{\infty}$. 
Hölder Spectrum of Typical Monotone Continuous Functions145

Since $f \in B\left(g_{\ell}, \varrho_{\ell}\right)$, we have

$$
|f(y)-f(x)| \geq\left|g_{\ell}(y)-g_{\ell}(x)\right|-2 \varrho_{\ell} \geq\left|g_{\ell}(y)-g_{\ell}(x)\right|-h_{\ell} / 4 .
$$

Recall that $g_{\ell}=g_{\ell}^{*}+g_{\ell}^{* *}$ and $g_{\ell}^{* *}$ increases by $h_{\ell}$ on each interval $\left[k / 2^{\nu \ell}, P_{\ell}(k)\right]$.

Assume $x \in F_{\infty} \subset F_{\ell}$ and $\alpha+\frac{1}{\ell}<\beta<1$. Then using $\delta_{\ell}<h_{\ell}^{\prime}$ there exists $y \in\left[x-2 h_{\ell}^{\prime}, x\right]$ such that $\left|g_{\ell}^{* *}(y)-g_{\ell}^{* *}(x)\right|=h_{\ell}$ and hence using (17) and (13) we obtain

$$
\begin{aligned}
|f(y)-f(x)| & \geq \frac{3}{4} h_{\ell} \geq \frac{3}{4}\left(\frac{h_{\ell}^{\prime}}{2^{\ell}}\right)^{\alpha} \geq \frac{3}{8} \frac{1}{\left(h_{\ell}^{\prime}\right)^{1 / \ell} 2^{\ell \alpha}}\left(2 h_{\ell}^{\prime}\right)^{\alpha+\frac{1}{\ell}} \\
& \geq \frac{3}{16} \frac{1}{h_{\ell}^{1 / \ell \alpha} 2^{\ell \alpha}}\left(2 h_{\ell}^{\prime}\right)^{\beta} \geq \frac{3}{16} \frac{1}{h_{\ell}^{1 / \ell \alpha} 2^{\ell \alpha}}|x-y|^{\beta}>|x-y|^{\beta}
\end{aligned}
$$

where the last inequality holds if we choose a sufficiently large $\ell$ and $\nu_{\ell}$, namely, choosing $\nu_{\ell}>m_{\ell}^{3} \geq \ell^{3}$ we have

$$
\frac{3}{16} \frac{1}{h_{\ell}^{1 / \ell \alpha} 2^{\ell \alpha}}=\frac{3}{16} \frac{1}{\left(h_{\ell}^{*}\right)^{1 / \ell \alpha}} 2^{\frac{\nu_{\ell}}{\ell \alpha}-\ell \alpha}>\frac{3}{16} 2^{\frac{\ell^{2}}{\alpha}-\ell \alpha}>1
$$

Since the above type estimations are valid for all $\beta \in(\alpha, 1)$ when $\ell$ is sufficiently large we obtain that $F_{\infty} \subset E_{\leq \alpha}^{f}$. From (16) and Proposition 1 it follows that $\operatorname{dim}_{H} F_{\infty} \geq \alpha$ which implies $\operatorname{dim}_{H}\left(E_{\leq \alpha}^{f}\right) \geq \alpha$.

Next we refine our argument to obtain $\operatorname{dim}_{H} E_{\alpha}^{f} \geq \alpha$. To obtain this result we find a subset $F_{\infty}^{0} \subset F_{\infty}$ for which $\mu_{\alpha}\left(F_{\infty}^{0}\right)>0$ and $F_{\infty}^{0} \subset E_{\alpha}^{f}$. By Proposition 1 this will imply the result about the Hausdorff dimension. To obtain $F_{\infty}^{0}$ we delete some components of $F_{\ell}$ to obtain the closed sets $F_{\ell}^{0}$. We will have a lower estimate on the $\mu_{\alpha}$ measures of these sets yielding $\mu_{\alpha}\left(F_{\infty}^{0}\right)=\mu_{\alpha}\left(\cap_{\ell=1}^{\infty} F_{\ell}^{0}\right)>0$. 


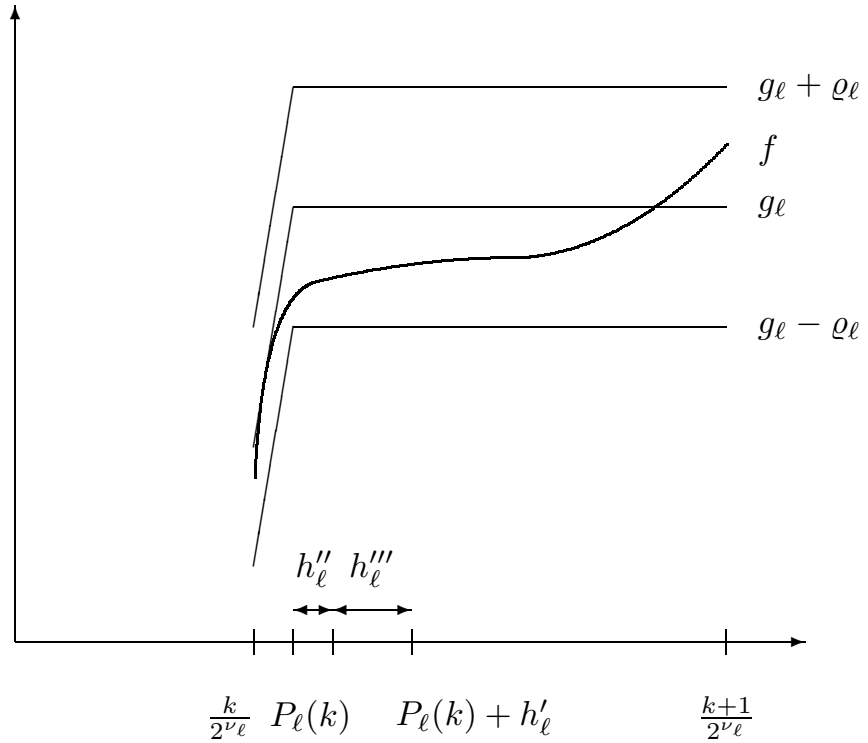

Assume $x \in F_{\ell}^{\prime}$. Then $x \in\left[P_{\ell}(k)+h_{\ell}^{\prime \prime}, P_{\ell}(k)+h_{\ell}^{\prime}\right] \subset\left[k / 2^{\nu_{\ell}},(k+1) / 2^{\nu_{\ell}}\right]$ for a suitable $k$. If $y \in\left[P_{\ell}(k),(k+1) / 2^{\nu_{\ell}}\right]$, then $g_{\ell}^{* *}(x)-g_{\ell}^{* *}(y)=0$. If $y \notin\left[P_{\ell}(k),(k+1) / 2^{\nu_{\ell}}\right]$, then $h_{\ell}^{\prime \prime} \leq|y-x|$ and one can easily see that

$$
\begin{aligned}
\left|g_{\ell}^{* *}(y)-g_{\ell}^{* *}(x)\right| & \leq\left(\left[\frac{|y-x|}{2^{-\nu_{\ell}}}\right]+1\right) h_{\ell} \leq h_{\ell}+|y-x| 2^{\nu_{\ell}} h_{\ell} \\
& =h_{\ell}+|y-x| h_{\ell}^{*}<\left(2 h_{\ell}^{\prime \prime}\right)^{\alpha}+|y-x| \\
& \leq 2^{\alpha}|y-x|^{\alpha}+|y-x| \leq 3|y-x|^{\alpha},
\end{aligned}
$$

where we also used (6) and (14).

In the next argument we assume that $\ell_{0}$ is sufficiently large and $\ell>\ell_{0}$. We define the sets $F_{\ell}^{0}$ by induction on $\ell$.

We assume that $\ell_{0}$ is so large that for all $\ell \geq \ell_{0}$ we have

$$
2 h_{\ell}^{\ell}<h_{\ell}^{\frac{1}{\alpha}+2}
$$

To start our induction we choose a component $J_{\ell_{0}} \stackrel{\text { def }}{=}\left[P_{\ell_{0}}\left(k^{\prime}\right)+h_{\ell_{0}}^{\prime \prime}, P_{\ell_{0}}\left(k^{\prime}\right)+h_{\ell_{0}}^{\prime}\right]$ of $F_{\ell_{0}}$ in a way that $g_{\ell_{0}}^{*}$ is constant on $J_{\ell_{0}}^{\prime} \stackrel{\text { def }}{=}\left[k^{\prime} / 2^{\nu \ell_{0}},\left(k^{\prime}+1\right) / 2^{\nu_{\ell_{0}}}\right]$ and set $F_{\ell_{0}}^{0}=J_{\ell_{0}}$. 
Hölder Spectrum of Typical Monotone Continuous Functions147

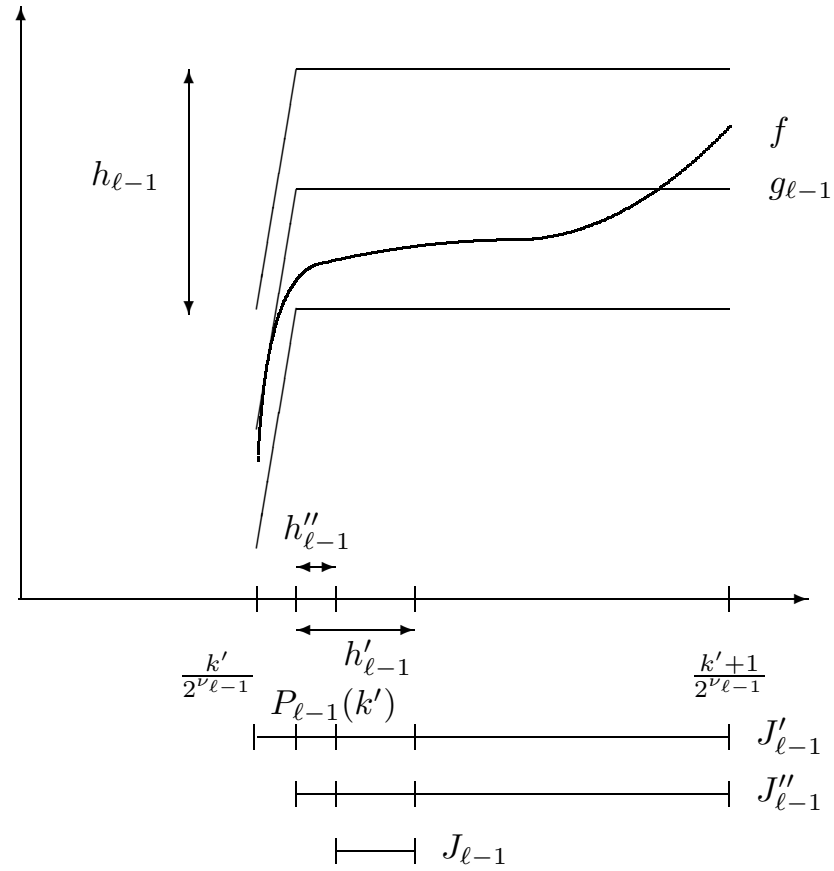

Assume $\ell>\ell_{0}$ and $J_{\ell-1} \stackrel{\text { def }}{=}\left[P_{\ell-1}\left(k^{\prime}\right)+h_{\ell-1}^{\prime \prime}, P_{\ell-1}\left(k^{\prime}\right)+h_{\ell-1}^{\prime}\right]$ is a component of $F_{\ell-1}^{0}$. Then $J_{\ell-1} \subset J_{\ell-1}^{\prime} \stackrel{\text { def }}{=}\left[k^{\prime} / 2^{\nu_{\ell-1}},\left(k^{\prime}+1\right) / 2^{\nu_{\ell-1}}\right]$. We also suppose that $g_{\ell-1}^{*}$ is constant on $J_{\ell-1}^{\prime}$. Set $J_{\ell-1}^{\prime \prime}=\left[P_{\ell-1}\left(k^{\prime}\right),\left(k^{\prime}+1\right) / 2^{\nu_{\ell-1}}\right]$. Observe that $g_{\ell-1}^{* *}$ is constant on $J_{\ell-1}^{\prime \prime}$; hence $g_{\ell-1}$ is also constant there. From $f \in B\left(g_{\ell-1}, \varrho_{\ell-1}\right)$ we infer

$$
f\left(\frac{k^{\prime}+1}{2^{\nu_{\ell-1}}}\right)-f\left(P_{\ell-1}\left(k^{\prime}\right)\right) \leq 2 \varrho_{\ell-1}=\frac{h_{\ell-1}^{m_{\ell-1}}}{4}<h_{\ell-1}^{\ell-1}<h_{\ell-1}^{\frac{1}{\alpha}+2}
$$

where we used (10), $m_{\ell} \geq \ell$ and (19).

From $f \in B\left(g_{\ell}, \varrho_{\ell}\right)$ and $2 \varrho_{\ell}<h_{\ell}^{\frac{1}{\alpha}+2}$ it follows that

$$
\left|g_{\ell}(y)-g_{\ell}(x)\right|-2 h_{\ell}^{\frac{1}{\alpha}+2} \leq|f(y)-f(x)| \leq\left|g_{\ell}(y)-g_{\ell}(x)\right|+2 h_{\ell}^{\frac{1}{\alpha}+2}
$$

for all $x, y$.

Assume $x \in J_{\ell-1} \subset F_{\ell-1}$ and $y \in\left[k^{\prime} / 2^{\nu_{\ell-1}}, P_{\ell-1}\left(k^{\prime}\right)\right]$. Then (18), (14), 


$$
\begin{aligned}
|y-x| \geq h_{\ell-1}^{\prime \prime} \text { and }\left|f-g_{\ell-1}\right| & \leq \varrho_{\ell-1}<h_{\ell-1}^{\frac{1}{\alpha}+2} \text { yield } \\
|f(y)-f(x)| & \leq 2 h_{\ell-1}^{\frac{1}{\alpha}+2}+\left|g_{\ell-1}(y)-g_{\ell-1}(x)\right| \\
& =2 h_{\ell-1}^{\frac{1}{\alpha}+2}+\left|g_{\ell-1}^{* *}(y)-g_{\ell-1}^{* *}(x)\right| \\
& \leq 2 h_{\ell-1}^{\frac{1}{\alpha}} h_{\ell-1}^{2}+3|y-x|^{\alpha} \\
& \leq 4 h_{\ell-1}^{\prime \prime} h_{\ell-1}^{2}+3|y-x|^{\alpha} \\
& \leq|y-x|+3|y-x|^{\alpha} \leq 4|y-x|^{\alpha} .
\end{aligned}
$$

Using (20) and (21) we obtain

$$
g_{\ell}\left(\frac{k^{\prime}+1}{2^{\nu_{\ell-1}}}\right)-g_{\ell}\left(P_{\ell-1}\left(k^{\prime}\right)\right) \leq h_{\ell-1}^{\frac{1}{\alpha}+2}+2 h_{\ell}^{\frac{1}{\alpha}+2}<2 h_{\ell-1}^{\frac{1}{\alpha}+2} .
$$

Since $g_{\ell}=g_{\ell}^{*}+g_{\ell}^{* *}$ and the functions $g_{\ell}^{*}, g_{\ell}^{* *}$ are monotone, we also have

$$
g_{\ell}^{*}\left(\frac{k^{\prime}+1}{2^{\nu_{\ell-1}}}\right)-g_{\ell}^{*}\left(P_{\ell-1}\left(k^{\prime}\right)\right)<2 h_{\ell-1}^{\frac{1}{\alpha}+2} \leq 4 h_{\ell-1}^{\prime \prime} h_{\ell-1}^{2}<\frac{1}{2} h_{\ell-1}^{\prime \prime \prime} h_{\ell-1}
$$

where we assumed that $\ell_{0} \leq \ell$ is sufficiently large and hence $4 h_{\ell-1}<1 / 2$.

Now Lemma 7 implies that there is an exceptional set $\Psi^{*}$ such that

$$
\lambda\left(\Psi^{*}\right)<2\left(g_{\ell}^{*}\left(\frac{k^{\prime}+1}{2^{\nu_{\ell-1}}}\right)-g_{\ell}^{*}\left(P_{\ell-1}\left(k^{\prime}\right)\right)\right)<h_{\ell-1}^{\prime \prime \prime} h_{\ell-1}
$$

and from $x \in J_{\ell-1}^{\prime \prime} \backslash \Psi^{*}$ it follows that $\left|g_{\ell}^{*}(y)-g_{\ell}^{*}(x)\right| \leq|y-x|$ holds for all $y \in J_{\ell-1}^{\prime \prime}$.

We can assume that $\nu_{\ell}$ is chosen so large that $2 / 2^{\nu_{\ell}}$ is less than all the jumps $g_{\ell}^{*}\left(\left(k^{\prime}+1\right) / 2^{N_{n}}\right)-g_{\ell}^{*}\left(k^{\prime} / 2^{N_{n}}\right)>0,\left(k^{\prime} \in\left\{0, \ldots, 2^{N_{n}}-1\right\}\right)$. Hence $g_{\ell}^{*}$ is either constant on an interval $\left[k / 2^{\nu_{\ell}},(k+1) / 2^{\nu_{\ell}}\right]$ or increases by at least $2 / 2^{\nu \ell}$.

This implies that $(b-a)>1 / 2^{\nu_{\ell}}$ for each component $(a, b)$ of $\Psi^{*}$. Denote by $\Psi_{0}^{*}$ the union of those intervals of the form $\left[k / 2^{\nu_{\ell}},(k+1) / 2^{\nu_{\ell}}\right]$ which intersect $\Psi^{*}$. By adding to each component $(a, b)$ of $\Psi^{*}$ those intervals $\left[k / 2^{\nu_{\ell}},(k+1) / 2^{\nu_{\ell}}\right]$ which contain $a$ and $b$ we obtain $\Psi_{0}^{*}$. Hence $\lambda\left(\Psi_{0}^{*}\right) \leq 3 \lambda\left(\Psi^{*}\right)<3 h_{\ell-1}^{\prime \prime \prime} h_{\ell-1}$. Denote by $F_{\ell}^{*}$ the union of those components of $F_{\ell} \cap J_{\ell-1}^{\prime \prime}$ which do not intersect $\Psi_{0}^{*}$. We define $F_{\ell}^{0}$ so that $F_{\ell}^{0} \cap J_{\ell-1}^{\prime \prime}$ equals $F_{\ell}^{*}$. Observe that $F_{\ell} \cap J_{\ell-1}=F_{\ell} \cap$ $J_{\ell-1}^{\prime}$ consists of $h_{\ell-1}^{\prime \prime \prime} 2^{\nu_{\ell}}$ many components and we need to delete $\lambda\left(\Psi_{0}^{*}\right) 2^{\nu_{\ell}}<$ $3 h_{\ell-1} h_{\ell-1}^{\prime \prime \prime} 2^{\nu_{\ell}}$ many of them to obtain $F_{\ell}^{0} \cap J_{\ell-1}^{\prime}=F_{\ell}^{0} \cap J_{\ell-1}$.

Since the $\mu_{\alpha}$ measure of each component of $F_{\ell}$ is $\mu_{\alpha}\left(F_{\ell} \cap J_{\ell-1}\right) 2^{-\nu_{\ell}} / h_{\ell-1}^{\prime \prime \prime}$, we obtain

$$
\mu_{\alpha}\left(F_{\ell}^{0} \cap J_{\ell-1}\right) \geq\left(1-3 h_{\ell-1}\right) \mu_{\alpha}\left(F_{\ell} \cap J_{\ell-1}\right)=\left(1-3 h_{\ell-1}\right) \mu_{\alpha}\left(F_{\ell-1}^{0} \cap J_{\ell-1}\right) .
$$


Hölder Spectrum of Typical Monotone Continuous Functions149

Adding the above inequalities for all $J_{\ell-1}$ type components of $F_{\ell-1}^{0}$ we have

$$
\mu_{\alpha}\left(F_{\ell}^{0}\right) \geq\left(1-3 h_{\ell-1}\right) \mu_{\alpha}\left(F_{\ell-1}^{0}\right) .
$$

Using $\nu_{\ell} \geq n_{\ell} \geq m_{\ell} \geq \ell$ and (6) we infer

$$
\mu_{\alpha}\left(F_{\infty}^{0}\right) \geq \prod_{\ell=\ell_{0}+1}^{\infty}\left(1-3 h_{\ell-1}\right) \mu_{\alpha}\left(F_{\ell_{0}}^{0}\right)>0 .
$$

For each $x \in F_{\ell}^{0}$ and $y \in J_{\ell-1}^{\prime \prime}$ we have $\left|g_{\ell}^{*}(y)-g_{\ell}^{*}(x)\right| \leq|y-x|$. We also know that $x \in F_{\ell}^{0} \subset F_{\ell}^{\prime}$ and (18) implies

$$
\left|g_{\ell}^{* *}(y)-g_{\ell}^{* *}(x)\right| \leq 3|y-x|^{\alpha} .
$$

Hence $\left|g_{\ell}(y)-g_{\ell}(x)\right| \leq 4|y-x|^{\alpha}$ holds for all $x \in F_{\ell}^{0}$ and $y \in J_{\ell-1}^{\prime \prime}$. By (21) we obtain $|f(y)-f(x)| \leq 4|y-x|^{\alpha}+2 h_{\ell}^{\frac{1}{\alpha}+2}$. We also note that if $J_{\ell} \stackrel{\text { def }}{=}\left[P_{\ell}(k)+\right.$ $\left.h_{\ell}^{\prime \prime}, P_{\ell}(k)+h_{\ell}^{\prime}\right]$ is a component of $F_{\ell}^{0}$, then $J_{\ell} \subset J_{\ell}^{\prime \text { def }}=\left[k / 2^{\nu_{\ell}},(k+1) / 2^{\nu_{\ell}}\right]$ and $g_{\ell}^{*}$ is constant on $J_{\ell}^{\prime}$, since otherwise $\left|g_{\ell}^{*}(y)-g_{\ell}^{*}(x)\right| \leq|y-x|$ cannot hold for all $y \in J_{\ell}^{\prime}$. (Recall that we assumed that the jumps of $g_{\ell}^{*}$ are at least $2 \cdot 2^{\nu_{\ell}}$.)

Assume $x \in F_{\ell}^{0}$ and $y \in J_{\ell-1}^{\prime \prime} \backslash J_{\ell}^{\prime}$. Then $|y-x| \geq h_{\ell}^{\prime \prime} \geq h_{\ell}^{1 / \alpha} / 2$; hence $2^{\alpha}|y-x|^{\alpha} \geq h_{\ell}$. Therefore

$$
|f(y)-f(x)| \leq 4|y-x|^{\alpha}+2 h_{\ell}^{\frac{1}{\alpha}+2} \leq 4|y-x|^{\alpha}+4 h_{\ell}^{2}|y-x| \leq 5|y-x|^{\alpha} .
$$

If $x \in F_{\ell}^{0} \subset F_{\ell-1}$ and $y \in\left[k^{\prime} / 2^{\nu_{\ell-1}}, P_{\ell-1}\left(k^{\prime}\right)\right)=J_{\ell-1}^{\prime} \backslash J_{\ell-1}^{\prime \prime}$, we have already seen in (22) that $|f(y)-f(x)| \leq 4|y-x|^{\alpha}$. Hence $|f(y)-f(x)| \leq 5|y-x|^{\alpha}$ holds for all $x \in F_{\ell}^{0}$ and $y \in J_{\ell-1}^{\prime} \backslash J_{\ell}^{\prime}$.

Finally, if $x \in \cap_{\ell=\ell_{0}}^{\infty} F_{\ell}^{0}=F_{\infty}^{0}$ and $x \neq y \in J_{\ell_{0}}^{\prime}$, then there exists $\ell \geq \ell_{0}+1$ such that $y \in J_{\ell-1}^{\prime} \backslash J_{\ell}^{\prime}$ and hence $|f(y)-f(x)| \leq 5|y-x|^{\alpha}$ holds. Therefore $F_{\infty}^{0} \subset E_{\alpha}^{f}$ and by Proposition 1, (25) and (16) we infer $\operatorname{dim}_{H}\left(F_{\infty}^{0}\right) \geq \alpha$.

This shows that for functions in $\mathcal{F}_{(i i)} \stackrel{\text { def }}{=} \mathcal{F}_{1}$ assertion (ii) holds.

\subsection{Assertion (iii)}

Proof. Assume $\mathcal{F}_{(i i i)}$ is the dense open set $\mathcal{F}^{*}$ of the main construction (used with the value of $h_{n, m}^{*}$ given in (6)) and $f \in \mathcal{F}_{(i i i)}$. Given $j_{0} \in \mathbb{N}$ set $m=j_{0}$ and choose $n \geq m$ such that $f \in G_{n, m}$. Then $N_{n} \geq K_{n}>n \geq m=j_{0}$; using (5) and the definition of $g_{n}^{*}$ we obtain

$$
\mu_{f_{n}, k^{\prime}} \stackrel{\text { def }}{=} \mu_{f_{n}}\left(\left[\frac{k^{\prime}}{2^{N_{n}}}, \frac{k^{\prime}+1}{2^{N_{n}}}\right]\right)=g_{n}^{*}\left(\frac{k^{\prime}+1}{2^{N_{n}}}\right)-g_{n}^{*}\left(\frac{k^{\prime}}{2^{N_{n}}}\right) .
$$


Since $f_{n}^{\prime}>0$ on $[0,1]$, we have $0<\mu_{f_{n}, \min } \stackrel{\text { def }}{=} \min \left\{\mu_{f_{n}, k^{\prime}}: k^{\prime}=0, \ldots, 2^{N_{n}}-\right.$ $1\}$ and we can assume that $\nu_{n, m}$ was chosen so large that $2^{-\nu_{n, m}}<\mu_{f_{n}, \min } / 8$; hence $h_{n, m}^{*}<2^{-\nu_{n, m}}<\mu_{f_{n}, \min } / 8$ and $\varrho_{n, m}<h_{n, m} / 8<h_{n, m}^{*} / 8<\mu_{f_{n}, \min } / 8$.

Recalling (8) we obtain

$$
\begin{aligned}
\mu_{f}\left(\left[\frac{k^{\prime}}{2^{N_{n}}}, \frac{k^{\prime}+1}{2^{N_{n}}}\right]\right) & =\left|f\left(\frac{k^{\prime}+1}{2^{N_{n}}}\right)-f\left(\frac{k^{\prime}}{2^{N_{n}}}\right)\right| \\
& \geq\left|g_{n, m}\left(\frac{k^{\prime}+1}{2^{N_{n}}}\right)-g_{n, m}\left(\frac{k^{\prime}}{2^{N_{n}}}\right)\right|-2 \varrho_{n, m} \\
& \geq\left|g_{n}^{*}\left(\frac{k^{\prime}+1}{2^{N_{n}}}\right)-g_{n}^{*}\left(\frac{k^{\prime}}{2^{N_{n}}}\right)\right|-2 \varrho_{n, m}-2 h_{n, m}^{*} \\
& >\frac{\mu_{f_{n}, k^{\prime}}}{2} .
\end{aligned}
$$

Thus, using (3) we have

$$
\tau_{f, N_{n}}(q) \geq \frac{1}{N_{n} \log 2} \log \sum_{k^{\prime}=0}^{2^{N_{n}}-1}\left(\mu_{f_{n}, k^{\prime}} / 2\right)^{q}=\tau_{f_{n}, N_{n}}(q)-\frac{q}{N_{n}} \geq 1-q-\frac{2}{j_{0}} .
$$

This implies $\bar{\tau}_{f}(q)=\lim \sup _{j \rightarrow \infty} \tau_{f, j}(q) \geq 1-q$. By Lemma 3 we obtain $\bar{\tau}_{f}(q)=1-q$.

Since $f$ is strictly monotone increasing, one can easily show that $\tau_{f}(0)=1$.

Assume $0<q \leq 1$ is given and fixed. We verify that $\underline{\tau}_{f}(q)=0$.

Given $f \in \mathcal{F}_{(i i i)}$ and $j_{0}>1 / q$ we choose $n, m$ as above. Observe that out of the $2^{\nu_{n, m}}$ intervals of the form $\left[k / 2^{\nu_{n, m}},(k+1) / 2^{\nu_{n, m}}\right],\left(k=0, \ldots, 2^{\nu_{n, m}}-1\right)$ there are only $2^{N_{n}}$ many intervals on which $g_{n}^{*}$ is nonconstant. We call these intervals irregular and the remaining intervals are called regular. We denote by $\Gamma$ the set of those $k \in\left\{0, \ldots, 2^{\nu_{n, m}}-1\right\}$ for which $\left[k / 2^{\nu_{n, m}},(k+1) / 2^{\nu_{n, m}}\right]$ is regular. For irregular $k$ 's we use the trivial estimate

$$
\left(\mu_{f}\left(\left[\frac{k}{2^{\nu_{n, m}}}, \frac{k+1}{2^{\nu_{n, m}}}\right]\right)\right)^{q} \leq \mu_{f}([0,1])=1
$$

where we used that replacing $f$ by $c f$ we can assume that $\mu_{f}$ is a probability measure. For $k \in \Gamma$ using $g_{n}^{*}\left((k+1) / 2^{\nu_{n, m}}\right)-g_{n}^{*}\left(k / 2^{\nu n, m}\right)=0$ we obtain

$$
\begin{aligned}
\mu_{f}\left(\left[\frac{k}{2^{\nu_{n, m}}}, \frac{k+1}{2^{\nu_{n, m}}}\right]\right) & =f\left(\frac{k+1}{2^{\nu_{n, m}}}\right)-f\left(\frac{k}{2^{\nu_{n, m}}}\right) \\
& \leq g_{n, m}\left(\frac{k+1}{2^{\nu_{n, m}}}\right)-g_{n, m}\left(\frac{k}{2^{\nu_{n, m}}}\right)+2 \varrho_{n, m}
\end{aligned}
$$




$$
\begin{aligned}
& =g_{n, m}^{* *}\left(\frac{k+1}{2^{\nu_{n, m}}}\right)-g_{n, m}^{* *}\left(\frac{k}{2^{\nu_{n, m}}}\right)+2 \varrho_{n, m} \\
& =h_{n, m}+2 \varrho_{n, m}<2 h_{n, m} .
\end{aligned}
$$

If $j_{0}$ and hence $n \geq m=j_{0}>1 / q$ is sufficiently large, then we can assume that $\nu_{n, m}>N_{n}^{2}$ satisfies $2^{\nu_{n, m}} \cdot 2^{q} h_{n, m}^{q}=2^{\nu_{n, m}} \cdot 2^{q}\left(h_{n, m}^{*} / 2^{\nu_{n, m}}\right)^{q}=2^{\nu_{n, m}}$. $2^{q}\left(1 / 4^{n} 2^{(m+1) \nu_{n, m}}\right)^{q} \leq 1<2^{N_{n}}$. Now using $\#\left(\left\{0, \ldots, 2^{\nu_{n, m}}-1\right\} \backslash \Gamma\right)=2^{N_{n}}$ $\# \Gamma<2^{\nu_{n, m}}$, and the above estimates we obtain

$$
\begin{aligned}
\tau_{f, \nu_{n, m}}(q) & \leq \frac{1}{\nu_{n, m} \log 2} \log \left(2^{N_{n}}+2^{\nu_{n, m}} \cdot 2^{q} h_{n, m}^{q}\right) \\
& \leq \frac{1}{\nu_{n, m} \log 2} \log \left(2 \cdot 2^{N_{n}}\right) \leq \frac{1}{\nu_{n, m} \log 2} \log \left(2^{\sqrt{\nu_{n, m}}}\right) \\
& \leq \frac{1}{\sqrt{\nu_{n, m}}} \leq \frac{1}{j_{0}},
\end{aligned}
$$

Hence $\underline{\tau}_{f}(q) \leq 0$ and Lemma 3 completes the proof showing that $\underline{\tau}_{f}(q)=0$.

\subsection{Assertion (iv)}

The proof of this assertion is similar at many places to that of Assertion (iii).

Proof. Assume $q>1$ is fixed. Let $\mathcal{F}_{(i v)}=\mathcal{F}^{*}$ of the main construction and for given $j_{0} \in \mathbb{N}$ choose $m, n$ and define $\mu_{f_{n}, k^{\prime}}, \mu_{f_{n}, \text { min }}$ as in the proof of Assertion (iii). We can again assume that $h_{n, m}^{*}<\mu_{f_{n}, \min } / 8$ and $\varrho_{n, m}<$ $\mu_{f_{n} \min } / 8$.

Then similarly to (26) we obtain

$$
\begin{aligned}
\mu_{f}\left(\left[\frac{k^{\prime}}{2^{N_{n}}}, \frac{k^{\prime}+1}{2^{N_{n}}}\right]\right) \leq & \left|g_{n}^{*}\left(\frac{k^{\prime}+1}{2^{N_{n}}}\right)-g_{n}^{*}\left(\frac{k^{\prime}}{2^{N_{n}}}\right)\right|+ \\
& 2 \varrho_{n, m}+2 h_{n, m}^{*}<2 \mu_{f_{n}, k^{\prime}} .
\end{aligned}
$$

We can use (4) when $n \geq j_{0} \geq q$ and we have

$$
\tau_{f, N_{n}}(q) \leq \frac{1}{N_{n} \log 2} \log \sum_{k^{\prime}=0}^{2^{N_{n}}-1}\left(2 \mu_{f_{n}, k^{\prime}}\right)^{q} \leq 1-q+\frac{q+1}{j_{0}} .
$$

This implies $\underline{\tau}_{f}(q) \leq 1-q$. By Lemma 5 we obtain $\underline{\tau}_{f}(q)=1-q$.

Next we show $\bar{\tau}_{f}(q) \geq 0$. 
Observe that from $\nu_{n, m}>N_{n}^{2}$ and (7) it follows that if we choose $j_{n}$ such that $2^{-j_{n}}=\delta_{n, m}$, then $\sqrt{j_{n}}>N_{n}$. We can also assume that $j_{0} \leq m \leq n$ is so large that

$$
g_{n}^{*}(1)-g_{n}^{*}(0) \geq f(1)-f(0)-2 \varrho_{n, m}-h_{n, m}^{*} \geq \frac{f(1)-f(0)}{2} .
$$

Observe that if $k^{\prime \prime} \in\left\{0, \ldots, 2^{j_{n}}-1\right\}$ is chosen so that $k^{\prime \prime} / 2^{j_{n}}=k^{\prime} / 2^{N_{n}}$ for a suitable $k^{\prime} \in\left\{0, \ldots, 2^{N_{n}}-1\right\}$, then

$$
g_{n}^{*}\left(\frac{k^{\prime \prime}+1}{2^{j_{n}}}\right)-g_{n}^{*}\left(\frac{k^{\prime \prime}}{2^{j_{n}}}\right)=\mu_{f_{n}, k^{\prime}},
$$

we denote the set of these $k^{\prime \prime}$ by $\Gamma^{\prime \prime}$.

For $k^{\prime \prime} \in \Gamma^{\prime \prime}$ similarly to (26) we obtain

$\mu_{f}\left(\left[\frac{k^{\prime \prime}}{2^{j_{n}}}, \frac{k^{\prime \prime}+1}{2^{j_{n}}}\right]\right) \geq g_{n}^{*}\left(\frac{k^{\prime}+1}{2^{N_{n}}}\right)-g_{n}^{*}\left(\frac{k^{\prime}}{2^{N_{n}}}\right)-2 \varrho_{n, m}-2 h_{n, m}^{*} \geq \frac{\mu_{f_{n}, k^{\prime}}}{2}$.

By using an inequality between means of non negative numbers we have

$$
\left(\frac{\sum_{k^{\prime}=0}^{2^{N_{n}}-1} \mu_{f_{n}, k^{\prime}}^{q}}{2^{N_{n}}}\right)^{\frac{1}{q}} \geq \frac{\sum_{k^{\prime}=0}^{2^{N_{n}}-1} \mu_{f_{n}, k^{\prime}}}{2^{N_{n}}}=\frac{g_{n}^{*}(1)-g_{n}^{*}(0)}{2^{N_{n}}} .
$$

Hence

$$
\sum_{k^{\prime}=0}^{2^{N_{n}}-1} \mu_{f_{n}, k^{\prime}}^{q} \geq\left(g_{n}^{*}(1)-g_{n}^{*}(0)\right)^{q} 2^{N_{n}(1-q)} \geq\left(\frac{f(1)-f(0)}{2}\right)^{q} 2^{N_{n}(1-q)} .
$$

Thus

$$
\begin{aligned}
\tau_{f, j_{n}}(q) & =\frac{1}{j_{n} \log 2} \log \sum_{k^{\prime \prime}=0}^{2^{j_{n}}-1}\left(\mu_{f}\left(\left[\frac{k^{\prime \prime}}{2^{j_{n}}}, \frac{k^{\prime \prime}+1}{2^{j_{n}}}\right]\right)\right)^{q} \\
& \geq \frac{1}{j_{n} \log 2} \log \sum_{k^{\prime \prime} \in \Gamma^{\prime \prime}}\left(\mu_{f}\left(\left[\frac{k^{\prime \prime}}{2^{j_{n}}}, \frac{k^{\prime \prime}+1}{2^{j_{n}}}\right]\right)\right)^{q} \\
& \geq \frac{1}{j_{n} \log 2} \log \left(\left(\frac{f(1)-f(0)}{2}\right)^{q} 2^{N_{n}(1-q)} \frac{1}{2^{q}}\right) \\
& \geq \frac{1}{j_{n} \log 2} \log \left(\left(\frac{f(1)-f(0)}{4}\right)^{q} 2^{\sqrt{j_{n}}(1-q)}\right) \\
& \geq \frac{1-q}{\sqrt{j_{n}}}+\frac{\log \left(\left(\frac{f(1)-f(0)}{4}\right)^{q}\right)}{j_{n} \log 2} .
\end{aligned}
$$

This implies $\bar{\tau}_{f}(q) \geq 0$. By Lemma 5 we obtain $\bar{\tau}_{f}(q)=0$. 


\subsection{Assertion (v)}

Proof. First we show $\underline{\tau}_{f}(q) \geq 1-q$. Assume $f \in \mathcal{F}_{(v)} \stackrel{\text { def }}{=} \mathcal{F}_{(i)}=\mathcal{F}_{1} \cap \mathcal{F}_{0}$. Then $f^{\prime}=0$ almost everywhere and hence there exists $\delta>0$ and a measurable set $H \subset[0,1]$ such that $\lambda(H)>1 / 2$ and for any $x \in H, y \in(x-\delta, x+\delta)$ we have

$$
|f(y)-f(x)|<|y-x| \text {. }
$$

Assume $j$ is chosen so that $1 / 2^{j}<\delta$. Denote by $\Gamma_{H}$ the set of those $k \in\left\{0, \ldots, 2^{j}-1\right\}$ for which $H \cap\left[k / 2^{j},(k+1) / 2^{j}\right] \neq \emptyset$. From $\lambda(H)>1 / 2$ it follows that $\# \Gamma_{H}>2^{j-1}$. From $(28)$ it follows that $\mu_{f}\left(\left[k / 2^{j},(k+1) / 2^{j}\right]\right)<2^{-j}$ holds when $k \in \Gamma_{H}$. Hence using $q<0$ we have

$$
\begin{aligned}
\tau_{f, j}(q) & \geq \frac{1}{j \log 2} \log \sum_{k \in \Gamma_{H}}\left(\mu_{f}\left(\left[\frac{k}{2^{j}}, \frac{k+1}{2^{j}}\right]\right)\right)^{q} \\
& >\frac{1}{j \log 2} \log \left(2^{j-1} 2^{-j q}\right)=\frac{j(1-q)-1}{j} .
\end{aligned}
$$

This implies $\underline{\tau}_{f}(q) \geq 1-q$.

Next we show $\underline{\tau}_{f}(q) \leq 1-q$. For any $n \geq m$ we have

$$
g_{n, m}\left(\frac{k+1}{2^{\nu_{n, m}}}\right)-g_{n, m}\left(\frac{k}{2^{\nu_{n, m}}}\right) \geq h_{n, m} .
$$

Since $\varrho_{n, m}<h_{n, m} / 8$ we have

$$
\begin{aligned}
\mu_{f, n, m, k} & =f\left(\frac{k+1}{2^{\nu_{n, m}}}\right)-f\left(\frac{k}{2^{\nu_{n, m}}}\right) \\
& >g_{n, m}\left(\frac{k+1}{2^{\nu_{n, m}}}\right)-g_{n, m}\left(\frac{k}{2^{\nu_{n, m}}}\right)-2 \varrho_{n, m} \\
& >\frac{3}{4} h_{n, m}=\frac{3}{4^{n+1} 2^{\nu_{n, m}}}>\frac{1}{4^{2 n} 2^{\nu_{n, m}}} .
\end{aligned}
$$

Keeping in mind that $q<0$ we infer

$$
\begin{aligned}
\tau_{f, \nu_{n, m}}(q) & =\frac{1}{\nu_{n, m} \log 2} \log \sum_{k=0}^{2^{\nu_{n}, m}-1} \mu_{f, n, m, k}^{q} \\
& <\frac{1}{\nu_{n, m} \log 2} \log \left(2^{\nu_{n, m}} 4^{-2 n q} 2^{-q \nu_{n, m}}\right)=(1-q)+\frac{-4 n q}{\nu_{n, m}} .
\end{aligned}
$$

From $\nu_{n, m}>N_{n}^{2} \geq K_{n}^{2}>n^{2} \geq m^{2}$ easily follows that $\frac{-4 n q}{\nu_{n, m}} \rightarrow 0$ as $m$, and hence $n$, tends to $\infty$. Therefore $\underline{\tau}_{f}(q) \leq 1-q$. 
Now we verify that $\bar{\tau}_{f}(q)=\infty$.

Observe that by (7), $P(k, n, m)<(2 k+1) / 2^{\nu_{n, m}+1}$ and hence $g_{n, m}$ is constant on $\left[(2 k+1) / 2^{\nu_{n, m}+1},(k+1) / 2^{\nu_{n, m}}\right]$. Therefore,

$$
f\left(\frac{k+1}{2^{\nu_{n, m}}}\right)-f\left(\frac{2 k+1}{2^{\nu_{n, m}+1}}\right)<2 \varrho_{n, m}=\frac{\left(h_{n, m}\right)^{m}}{4} .
$$

Thus in line (29) below recalling (11) we obtain

$$
\begin{aligned}
\tau_{f, \nu_{n, m}+1}(q) & =\frac{1}{\left(\nu_{n, m}+1\right) \log 2} \log \sum_{k^{\prime}=0}^{2^{\nu_{n, m}+1}-1}\left(f\left(\frac{k^{\prime}+1}{2^{\nu_{n, m}+1}}\right)-f\left(\frac{k^{\prime}}{2^{\nu_{n, m}+1}}\right)\right)^{q} \\
& >\frac{1}{\left(\nu_{n, m}+1\right) \log 2} \log \sum_{k=0}^{2^{\nu_{n, m}}-1}\left(f\left(\frac{k+1}{2^{\nu_{n, m}}}\right)-f\left(\frac{2 k+1}{2^{\nu_{n, m}+1}}\right)\right)^{q} \\
& >\frac{1}{\left(\nu_{n, m}+1\right) \log 2} \log \left(2^{\nu_{n, m}} \frac{\left(h_{n, m}\right)^{m q}}{4^{q}}\right) \\
& =\frac{1}{\left(\nu_{n, m}+1\right) \log 2} \log \left(2^{\nu_{n, m}}\left(4^{n} 2^{\nu_{n, m}}\right)^{-m q} 4^{-q}\right) \\
& =\frac{\nu_{n, m}(1-m q)}{\nu_{n, m}+1}+\frac{-2 m n q \log 2+\log \left(4^{-q}\right)}{\left(\nu_{n, m}+1\right) \log 2} .
\end{aligned}
$$

Recalling that $q<0$ and $\nu_{n, m}>N_{n}^{2} \geq K_{n}^{2}>n^{2} \geq m^{2}$ we obtain $\bar{\tau}_{f}(q)=\infty$.

\subsection{Assertion (vi)}

Assume $f \in \mathcal{F}_{(v i)} \stackrel{\text { def }}{=} \mathcal{F}_{(i i)}$. We will use the notation and choice of $h_{n, m}^{*}$ introduced in the proof of assertion (ii) and hence choose a suitable subsequence $m_{\ell} \geq \ell$. We will denote $W_{\ell}=W_{n\left(m_{\ell}\right), m_{\ell}}$. Clearly,

$$
\begin{aligned}
\mu_{g_{\ell}}\left(W_{\ell}\right) & =\sum_{k=0}^{2^{\nu_{\ell}}-1} g_{\ell}\left(P_{\ell}(k)\right)-g_{\ell}\left(\frac{k}{2^{\nu_{\ell}}}\right)=\sum_{k=0}^{2^{\nu_{\ell}}-1} g_{\ell}\left(\frac{k+1}{2^{\nu_{\ell}}}\right)-g_{\ell}\left(\frac{k}{2^{\nu_{\ell}}}\right) \\
& =g_{\ell}(1)-g_{\ell}(0) \geq f(1)-f(0)-2 \varrho \ell .
\end{aligned}
$$

Assuming $m_{\ell} \geq 2$, we have by (6) and (10) $\varrho_{\ell}<h_{\ell}^{2} / 2<h_{\ell} / 2^{\nu_{\ell}+1}$. There- 
fore

$$
\begin{aligned}
\mu_{f}\left(W_{\ell}\right) & =\sum_{k=0}^{2^{\nu_{\ell}}-1} f\left(P_{\ell}(k)\right)-f\left(\frac{k}{2^{\nu_{\ell}}}\right) \\
& \geq \sum_{k=0}^{2^{\nu_{\ell}}-1}\left(g_{\ell}\left(P_{\ell}(k)\right)-g_{\ell}\left(\frac{k}{2^{\nu_{\ell}}}\right)-2 \varrho_{\ell}\right) \\
& \geq f(1)-f(0)-2^{\nu_{\ell}+1} \varrho_{\ell} \\
& >f(1)-f(0)-h_{\ell}>f(1)-f(0)-\frac{1}{2^{\ell}} .
\end{aligned}
$$

Thus $\mu_{f}\left([0,1] \backslash W_{\ell}\right)<1 / 2^{\ell}$. By the Borel-Cantelli lemma $\mu_{f}$-almost every $x \in[0,1]$ belongs to finitely many of the sets $[0,1] \backslash W_{\ell}$; that is, for $\mu_{f}$ - almost every $x$ there exists $\ell_{x}$ such that for all $\ell \geq \ell_{x}$ we have $x \in W_{\ell}$.

If $x \in W_{\ell}$; that is, $x \in\left(k / 2^{\nu_{\ell}}, P_{\ell}(k)\right)$ for a suitable $k$, then

$$
\begin{aligned}
f\left(P_{\ell}(k)\right)-f\left(\frac{k}{2^{\nu_{\ell}}}\right) & \geq g_{\ell}\left(P_{\ell}(k)\right)-g_{\ell}\left(\frac{k}{2^{\nu_{\ell}}}\right)-2 \varrho_{\ell} \\
& \geq g_{\ell}^{* *}\left(P_{\ell}(k)\right)-g_{\ell}^{* *}\left(\frac{k}{2^{\nu_{\ell}}}\right)-2 \varrho_{\ell}=h_{\ell}-2 \varrho_{\ell} \\
& >\frac{h_{\ell}}{2}>\frac{\delta_{\ell}^{\frac{1}{2 m_{\ell}}}}{2}>\frac{\delta_{\ell}^{\frac{1}{2 \ell}}}{2} .
\end{aligned}
$$

Taking $y=k / 2^{\nu_{\ell}}$ or $y=P_{\ell}(k)$ we have

$$
|f(y)-f(x)| \geq \frac{f\left(P_{\ell}(k)\right)-f\left(k / 2^{\nu \ell}\right)}{2}>\frac{\delta_{\ell}^{\frac{1}{2 \ell}}}{4} .
$$

On the other hand $|y-x|<\delta_{\ell}$ and hence $|f(y)-f(x)|>|y-x|^{1 / 2 \ell} / 4$. Since we can choose any $\ell \geq \ell_{x}$, we have $h_{f}(x)=0$. This shows $h_{f}=0$, $\mu_{f}$-almost everywhere in $[0,1]$; that is, $\mu_{f}\left(E_{\leq 0}^{f}\right)=f(1)-f(0)=\mu_{f}([0,1])$.

\section{References}

[1] G. Brown, G. Michon and J. Peyrière, On the multifractal analysis of measures, J. Statist. Phys. 66 no. 3-4 (1992), 775-790.

[2] S. Jaffard,Multifractal formalism for functions Part I: Results valid for all functions, SIAM J. Math. Anal, 28 No. 4 (1997), 944-970.

[3] S. Jaffard, Multifractal formalism for functions Part II: Self-similar functions, SIAM J. Math. Anal, 28 No. 4 (1997), 971-998. 
[4] S. Jaffard, The spectrum of singularities of Riemann's function, Rev. Mat. Iberoamericana, 12 (1996) 441-460.

[5] S. Jaffard, Old friends revisited: the multifractal nature of some classical functions, J. of Fourier Anal. and Appl. 3 No. 1 (1997), 1-22.

[6] K. Falconer, Fractal Geometry, John Wiley \& Sons (1990).

[7] K. Falconer, Techniques in Fractal Geometry, John Wiley \& Sons (1997).

[8] H. Federer, Geometric Measure Theory, Springer Verlag, New York, 1969.

[9] J. Genyuk, A typical measure typically has no local dimension, Real Anal. Exchange, 23(2), 1997/8, 525-538.

[10] H. Haase, A survey on the dimension of measures, Topology, measures, and fractals (Warnemünde, 1991), 66-75, Math. Res., 66, AkademieVerlag, Berlin 1992.

[11] T. Zamfirescu, Most monotone functions are singular, Amer. Math. Monthly, 88 (1) (1981), 47-49.

[12] T. Zamfirescu, Typical monotone continuous functions, Arch. Math., 42 (1984), 151-156. 